\title{
Zukunftsgestaltung konkret
}

\section{Der Ressourcenverbrauch für das Baven und Wohnen ist nicht nachhaltig. Be- dürfnisfeldbezogene Szenarien zeigen Handlungspotenziale und deren Grenzen auf. Das Beispiel Schleswig-Holstein zeigt, wie mithilfe eines solchen metho- dischen Vorgehens ein Umsteuerungsprozess in Gang gesetzt werden kann, wenn alle relevanten Akteure einbezogen werden.}

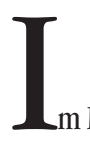
Von K. O. Henseling, K.-D. Sturm und M. Vallenthin steht die Einsicht, dass soziale Verantwortung, wirtschaftliche Leistungsfähigkeit und der Schutz der natürlichen Umwelt untrennbar zusammengehören. Nur innerhalb des Spielraumes, den die Natur als Lebensgrundlage bereitstellt, kann auf Dauer sozial verträglich gewirtschaftet werden. Aufgabe der Politik ist es, durch die Festlegung von Umweltzielen und die Vorgabe von rechtlichen und ökonomischen Rahmenbedingungen die Fahrrinne vorzugeben, die das Schiff der wirtschaftlichen und sozialen Entwicklung beachten muss, wenn die Lebensgrundlagen kommender Generationen nicht geschädigt werden sollen.

Ressourcenschonung als Gebot der Nachhaltigkeit ist - anders als klassische Ziele der Umweltpolitik - nicht allein durch technische Regeln oder Verbote zu erreichen. Hier ist eine konstruktiv-gestaltende Politik gefordert, die zugleich auf anhaltende und langfristige Mitgestaltung von allen gesellschaftlichen Akteuren ausgerichtet ist.

Zur Unterstïtzung dieses Prozesses dient der Bedürfnisfeldansatz. Kern dieses Ansatzes ist die Erkenntnis, dass Bedürfnisse wirtschaftliche Aktivitäten in Gang setzen und damit Stoffströme und Umweltbelastungen verursachen. Die Bedürfnisse der Menschen lassen sich in Bedürfnisfelder wie Wohnen, Mobilität, Ernährung, Information und Bildung zusammenfassen. Unterschiedlichen Varianten und Entwicklungen der Bedürfnisbefriedigung entsprechen unterschiedliche Muster der Umweltinanspruchnahme, die in Szenarien abbildbar sind und mit gesamtgesellschaftlichen Zielvorgaben in Beziehung gesetzt werden können. Eine Nachhaltigkeitsstrategie für ein Bedürfnisfeld muss die Entwicklungspfade herausfinden und unterstützen, die sich in der - durch Umweltziele bestimmten - „Fahrrinne“ der Trag- und Leistungsfähigkeit des Naturhaushaltes befinden.

\section{- Bedürfnisfeld Baven und Wohnen}

Für das Thema Ressourcenschonung ist das Bedürfnisfeld Bauen und Wohnen von besonders hoher Bedeutung. Baurohstoffe, wie Kies und Sand, haben einen wesentlichen Anteil an den Stoffmengenströmen in Deutschland. Auch am Verbrauch des nachwachsenden Rohstoffs Holz ist der Bausektor in erheblichem Umfang beteiligt. Durch die Ausweisung von Bauland findet eine zunehmende Flächeninanspruchnahme statt. Auch ein erheblicher Teil der in Deutschland verwendeten Energie wird im Bedürfnisfeld Bauen und Wohnen in Anspruch genommen. In allen diesen Bereichen stößt eine zunehmende Ressourceninanspruchnahme an Grenzen: Beim Abbau von Baurohstoffen verschärfen sich Nutzungskonflikte. Eine weiter zunehmende Flächeninanspruchnahme steht im Widerspruch zu dem Erfordernis der Erhaltung von naturnahen Gebieten und dem Schutz der biologischen Vielfalt. Die Verringerung der verwendeten Energiemenge ist insbesondere aus Gründen des Klimaschutzes dringend erforderlich. Um zu einer nachhaltigen Entwicklung zu gelangen, muss die Entwicklung der Umweltinanspruchnahme in diesem Bedürfnisfeld in eine Richtung gelenkt werden, die den Erfordernissen einer nachhaltigen Nutzung der natïrlichen Ressourcen entspricht.

Durch die Erstellung von Szenarien können Umweltentlastungspotenziale unterschiedlichen Handlungsoptionen zugeordnet werden. Um realistische Handlungsspielräume in dem Bedürfnisfeld ermitteln zu können, ist die aktive Beteiligung der in dem Bedürfnisfeld handelnden Akteure erforderlich. Im Auftrag des Umweltbundesamtes hat das Öko-Institut detaillierte und umfassende Stoffstromanalysen für das Bedürfnisfeld Bauen und Wohnen durchgeführt. Neben einer belastbaren Datenbasis für die Bundesebene wurde eine adäquate Methodik und ein Software-Tool (BASiS = Bedarfsorientiertes
Analysewerkzeug für Stoffströme in Szenarien) für die Berechnung von Szenarien im Bedürfnisfeld Bauen und Wohnen geschaffen (1).

Mit dieser Software wurden auf Bundesebene und in dem Projekt „Nachhaltiges Bauen und Wohnen in Schleswig-Holstein" (2) Szenarioberechnungen durchgefuihrt. Dabei wurde einem Referenz-Szenario jeweils ein Nachhaltigkeits-Szenario gegenüberstellt, bei dem unter anderem angenommen wurde, dass der Gebäudebestand besser genutzt wird, verstärkt Mehrfamilienhäuser und weniger Ein- und Zweifamilienhäuser gebaut werden, die Ansprüche an die Haus- und Wohnungsgröße etwas bescheidener werden, der Anteil an Niedrigenergie- und Passivhäusern stärker zunimmt, mehr mit Holz gebaut wird, der Bestand zïgig wärmetechnisch saniert wird, effizientere Heizungsanlagen eingebaut und bei der Herstellung von Baustoffen effizientere Verfahren angewendet werden.

\section{Ergebnisse oder Szenarien}

Die Ergebnisse der Nachhaltigkeits-Szenarien zeigen beispielsweise auf, welche Energieeinsparpotenziale im Bedürfnisfeld Bauen und Wohnen bestehen, die zur Erfüllung des Umwelthandlungszieles der Bundesregierung, die in Deutschland emittierte Kohlendioxidmenge bis 2005 um 25 Prozent gegenüber dem Stand von 1990 zu senken, beitragen können. Es wird deutlich, dass bis 2005 im Bedürfnisfeld Bauen und Wohnen unter den getroffenen Szenarioannahmen eine weitaus höhere prozentuale Kohlendioxidemissionsminderung erreichbar wäre und bis 2020 eine Halbierung der Kohlendioxidemissionen erreicht werden könnte. Das umweltpolitische Ziel, die zusätzliche Flächeninanspruchnahme für Siedlung und Verkehr drastisch zu reduzieren, kann dagegen unter den getroffenen Annahmen - die nicht von umweltpolitischen Erfordernissen, sondern von derzeit realisierbar erscheinenden Umorientierungen ausgingen - nicht erreicht werden. Dieses Ziel hat bisher noch zu wenig Resonanz im öffentlichen Bewusstsein gefunden.

Um die Entwicklung im Bedürfnisfeld Bauen und Wohnen in allen wichtigen Bereichen in die Fahrrinne dauerhaft umweltverträglichen Wirtschaftens lenken zu können, sind also Veränderungen erforderlich, die über das hinausgehen, was für den Szenariozeitraum der nächsten 20 Jahre unter den optimistischen Annahmen eines NachhaltigkeitsSzenarios für realisierbar gehalten wird. Mit dem Bedürfnisfeldansatz und der Szenariomethode kann auch gezeigt werden, in welchen Bereichen 
die Nachhaltigkeitspolitik Ziele bereits in absehbarer Zeit erreichen kann und wo noch besonders dicke Bretter gebohrt werden müssen.

Das Stoffstrommodell BASiS wird derzeit im Auftrag des Umweltbundesamtes um den Bereich der technischen Infrastruktur (Wohnstraßen, Verund Entsorgung) erweitert (3). Mit dem erweiterten Modell berechnete Szenarien fließen in ein weiteres Projekt „Kriterien und Indikatorensysteme für ein nachhaltig umweltverträgliches Bauen und Wohnen“ ein (4).

\section{- Das Beispiel Schleswig-Holstein}

Aufgrund der aufgezeigten überragenden Bedeutung ist das Bedürfnisfeld Bauen und Wohnen ein wichtiger Baustein des Projektes ,Zukunftsfähiges Schleswig-Holstein“. Von Beginn des Projektes an hat das Umweltministerium großen Wert darauf gelegt, dass die relevanten Akteure in das Vorhaben eingebunden sind: die Wohnungsbaugesellschaften, die Bauwirtschaft, die Investitionsbank SchleswigHolstein, der Mieterverein, die Umweltverbände, die Architekten- und Ingenieurkammer SchleswigHolstein, die Arbeitsgemeinschaft für zeitgemäßes Bauen und das Innenministerium als oberste schleswig-holsteinische Landesbehörde für Wohnungs- und Städtebau. Dies ist eine wichtige Voraussetzung für die kooperative Erarbeitung einer Nachhaltigkeitsstrategie.

In einem ersten Arbeitsschritt wurden die Akteure mit den Zielen und der Methode des Projektes vertraut gemacht. In direktem Kontakt mit den unterschiedlichen Akteuren haben die Projektbearbeiter die in BASiS vorliegenden nationalen Daten an die spezifische Situation in SchleswigHolstein angepasst. Damit stand eine an die realen Verhältnisse des Ist-Zustandes (Basisjahr 1998) angeglichene Datenbasis zur Verfügung. In der zweiten Projektphase wurden zusammen mit den Akteuren in einem intensiven Arbeitsprozess Szenarioannahmen für das Referenz-Szenario und

\section{Einen Überblick über alle bisher erschienenen Hefte des Informationsdienstes "Ökologisches Wirtschaften" \\ finden Sie auf unseren Internetseiten unter der Adresse:}

\section{http://www.oekom.de}

das Nachhaltigkeits-Szenario für das Bauen und Wohnen in Schleswig-Holstein bis zum Jahr 2020 entwickelt und intensiv diskutiert.

Die Vorgehensweise wird an zwei Beispielen kurz erläutert:

- Aufgrund vorliegender Prognosen wurde im Referenz-Szenario für den Abgang alten Wohnungsbestandes ein erheblicher Anstieg (Verzehnfachung der Abgangsquote bis 2005) angenommen. Für das Nachhaltigkeits-Szenario nahmen die Akteure an, dass ein solcher Anstieg durch eine forcierte Bestandserhaltungspolitik vermieden werden kann und dadurch auch ein (um ein Drittel) geringerer Zubau erforderlich wird.

- Für die Holzbauweise wurde im Referenz-Szenario ein konstanter Anteil auf dem derzeitigen Niveau (10 bis 15 Prozent) zugrunde gelegt. Für das Nachhaltigkeits-Szenario einigten sich die Akteure nach lebhafter, zum Teil kontroverser Diskussion auf eine Verdoppelung des Marktanteils für die Holzbauweise auf 20 bis 30 Prozent bis zum Jahr 2020.

In der dritten Arbeitsphase wurden die mit Hilfe der Szenariotechnik aufgedeckten Nachhaltigkeits-Potenziale visualisiert. In einem Workshop wurden auf der Basis der Ergebnisse der Szenarioberechnungen - die sich in ihren Aussagen deutlich unterscheiden - Handlungsoptionen identifiziert und Prioritäten für eine vorsorgende Umweltpolitik zusammen mit den Akteuren herausgearbeitet. Darauf folgte eine Diskussion möglicher und notwendiger Maßnahmen für ein nachhaltiges Bauen und Wohnen in SchleswigHolstein, die von der Neuorientierung von Förderprogrammen bis zu Novellierungsvorschlägen für die Landesbauordnung reichen. Dabei kommt der Altbausanierung in den Städten sowohl aus ökologischen Gründen als auch unter ökonomischen und beschäftigungspolitischen Zielsetzungen eine besondere Bedeutung zu.

\section{Schritte zur Umsetzung}

Die Ergebnisse zeigen, dass Schleswig-Holstein in dem Bedürfnisfeld Bauen und Wohnen bereits einige wichtige Weichen für eine Nachhaltige Entwicklung gestellt hat. Dazu zählen die baurechtliche Gleichstellung der Holzbauweise mit der Massivbauweise im Rahmen der Novellierung der Landesbauordnung und das Impulsprogramm „Wärmetechnische Gebäudesanierung“. Die Fraktionen von SPD und Bündnis 90/Die Grünen haben die Ergebnisse des Projektes in ihrem Antrag vom 27.09.2000 „Zukunft des Wohnungsbaus" aufgegriffen. Darin bittet der
Landtag die Landesregierung im Wohnungsprogramm 2001-2002 „Belange des flächen- und ressourcensparenden Bauens, des Klimaschutzes und des Energiesparens - wie eine stärkere Ausrichtung auf wärmetechnische Altbausanierung und Passivhauskonzepte in den allgemeinen Förderkriterien stärker als bisher zu berücksichtigen".

Im nächsten Schritt des Projektes sollen in einem breit angelegten Dialog- und Verständigungsprozess zusammen mit den relevanten Akteuren die für ein nachhaltiges Bauen und Wohnen in Schleswig-Holstein notwendigen Maßnahmen entwickelt und dann auch umgesetzt werden.

\section{Anmerkungen}

(1) Umweltbundesamt (Hrsg.): Stoffflussbezogene Bausteine für ein nationales Konzept der Nachhaltigen Entwicklung. UBA-Texte 47/99. Berlin 1999. Die Broschüre zum Projekt „Bedürfnisse und Stoffströme - Bauen und Wohnen“ ist kostenlos erhältlich über: Umweltbundesamt, ZAD, Postfach 330022, 14191 Berlin

(2) Ministerium für Umwelt, Natur und Forsten, SchleswigHolstein (Hrsg.): Nachhaltiges Bauen \& Wohnen in Schleswig-Holstein. Kiel 2000. Das Projekt wurde ab 1999 vom Öko-Institut durchgeführt. Der Projektbericht ist kostenlos über Dr. K.-Dietrich Sturm erhältlich (Adresse siehe unten). (3) Das Projekt "Stoffflussbezogene Bausteine für ein nationales Konzept der Nachhaltigen Entwicklung - Verknüpfung des Bereichs Bauen und Wohnen mit dem komplementären Bereich Öffentliche Infrastruktur" wird vom Öko-Institư zusammen mit dem Institut für ökologische Raumentwicklung Dresden durchgeführt.

(4) Das Projekt wird im Auftrag des Umweltbundesamtes von einer Arbeitsgemeinschaft durchgeführt, die vom Institu† Wohnen und Umwelt GmbH geleitet wird und der das Institut für ökologische Raumentwicklung, das Öko-Institut und der Lehrstuhl Ökonomie und Ökologie des Wohnungsbaus der Universität Karlsruhe angehören.

\section{Die Autoren}

Dr. Karl Otto Henseling ist wissenschaftlicher Rat, Mark Vallenthin technischer Angestellter im Fachgebiet III 1.1 des Umweltbundesamtes, Arbeitsschwerpunkt Stoffstrommanagement.

Kontakt: Seecktstr. 6-10, 13581 Berlin;

Tel. 030/8903-3593, Fax -3232,

E-mail: karl-otto.henseling@uba.de, mark.vallenthin@uba.de

Dr. K.-Dietrich Sturm ist Referatsleiter im Ministerium für Umwelt, Natur und Forsten

Schleswig-Holstein (MUNF).

Kontakt: MUNF, Ref. V 12, Mercatorstraße 1-3, 24106 Kiel. E-mail: klaus-dietrich.sturm@umin.landsh.de 
(c) 20I0 Authors; licensee IÖW and oekom verlag. This is an article distributed under the terms of the Creative Commons Attribution Non-Commercial No Derivates License (http://creativecommons.org/licenses/by-nc-nd/3.o/), which permits unrestricted use, distribution, and reproduction in any medium, provided the original work is properly cited. 